\title{
Anticholinergic delirium superimposed on severe cognitive deficit secondary to benzhexol and flupenthixol decanoate: A dilemma in management
}

\author{
Siti Nor Fadhlina Misron ${ }^{1^{*}}$, Zahiruddin Othman $^{2}$, Lukmanul Hakim Misron ${ }^{3}$ \\ ${ }^{1}$ Department of Psychiatry, Hospital Tawau, Peti Surat 67, Tawau, Sabah, Malaysia \\ ${ }^{2}$ Department of Psychiatry, School of Medical Sciences, Universiti Sains Malaysia, Kubang Kerian, Kota Bharu, \\ Kelantan, Malaysia \\ ${ }^{3}$ Department of Anaesthesiology, Hospital Sultanah Aminah, Jalan Persiaran Abu Bakar Sultan, Johor Babru, Johor, \\ Malaysia
}

\begin{abstract}
The first and second generation antipsychotics are associated with cognitive impairment through the antidopaminergic and anticholinergic effects. However serious anticholinergic adverse events may occur in susceptible individual, such as being illustrated in this case, even though flupenthixol decanoate has never been reported to cause them. The concomitant use of anticholinergic medication to counter the antidopaminergic side effects may further accentu ate this condition and leads to delirium despite within the therapeutic dosage.
\end{abstract}

Key Words: Anticholinergic delirium, cognitive deficit, flupenthixol decanoate, benzhexol

\section{Introduction}

Schizophrenia is associated with cognitive deficit either due to the disease itself or from the side effects of medication. Cognition mainly the executive function is under the jurisdiction of prefrontal cortex via the influence of both dopamine and acetylcholine neurotransmitters $(1,2)$. Antipsychotics are the notorious cause of cognitive deficit via two mechanisms which are $\mathrm{D}_{4}$ and/or cholinergic receptors blockade. Antipsychotics dosage was negatively correlated with the cognition particularly on the declarative memory (3). A study showed that the cognition of schizophrenia patient is generally impaired with the increment of the antipsychotics dosage with or without anticholinergic property. But, those treated with antipsychotics that possess anticholinergic property fare even worse (4). However, anticholinergic delirium associated with flupenthixol and benzhexol within therapeutic dosages have never been reported. Thereby, it is crucial to be vigilant of this serious adverse effect to accurately identify and manage the condition appropriately.

\section{Case Report}

This 59-year-old man was being treated for paranoid schizophrenia. He was on oral haloperidol $15 \mathrm{mg}$ omni nocte, oral benzhexol $2 \mathrm{mg}$ bis in die, and intramuscular flupenthixol decanoate $60 \mathrm{mg}$ monthly. After two months of treatment, his wife noted that the patient gradually became forgetful. Concurrently, he exhibited signs of parkinsonism such as tremor, bradykinesia, and rigidity of the limbs. The haloperidol was tapered off with the impression that the extrapyramidal and cognitive side effects were secondary to excessive blockade of dopamine receptors. The parkinsonism completely abated after the increment of benzhexol to $2 \mathrm{mg}$ omni mane and $4 \mathrm{mg}$ omni nocte. However, he became disorientated to time, place, and person indicating a delirium. He became dependant on his wife for the activity of daily living. He ate less and loss $5 \mathrm{~kg}$ weight within a month. He also had constipation. Physical examination showed dry skin and mouth, normal temperature and stable vital signs. The Elderly Cognitive Assessment Questionnaire (ECAQ) score was $3 / 11$. He could not perform the written 


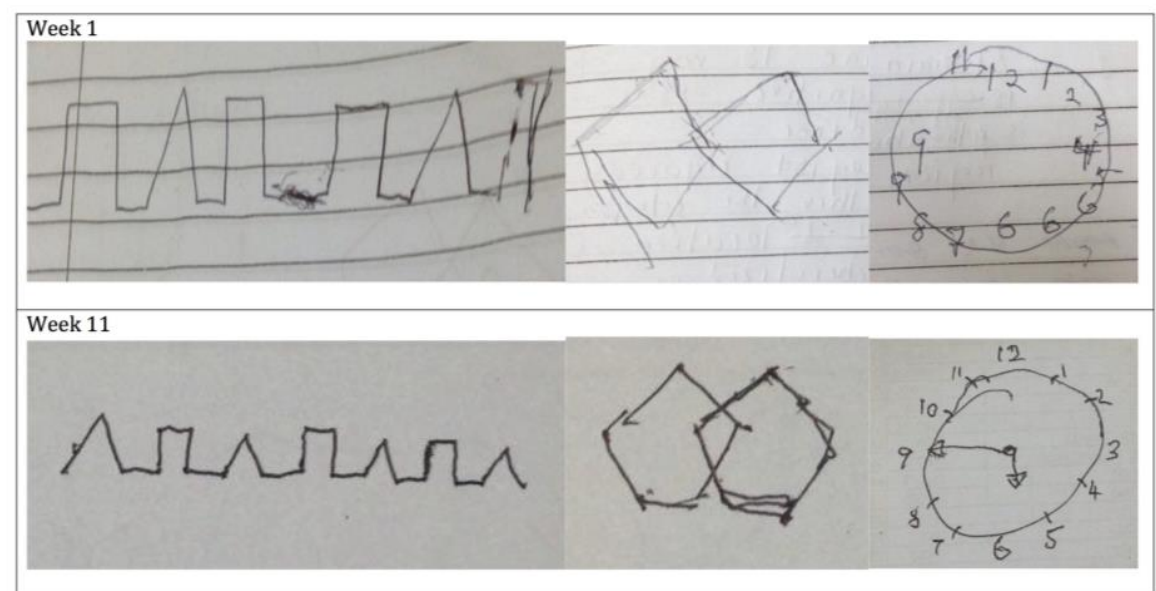

Fig. 1. Comparison of written alternating sequencing task test, pentagons test, and clock drawing test at one week (above) and 11 weeks (below) post Flupenthioxol Decanoate injection.

alternating sequencing task test, pentagons test, and clock drawing test. The full blood count, renal profile, liver function test, and thyroid function test were all within normal range. Mantoux test and chest X-ray excluded tuberculosis. Brain CT was normal. There were no other signs or symptoms suggestive of infection during this time.

Intramuscular procyclidine $10 \mathrm{mg}$ temporarily improved his cognition (ECAQ 5/11) but soon deteriorated again (ECAQ 1/11). All the medications were stopped and he was treated as an in-patient. There was no further recurrence of extrapyramidal side effect despite the termination of benzhexol. Adequate hydration and abstinence from any drugs with anticholinergic and/or antidopaminergic action was ensured. The delirium resolved in 3 weeks following the termination of oral medication or 4 weeks after the last flupenthixol decanoate administration. The constipation was resolving but the dry skin and mouth were still present at that time. Nevertheless, the cognitive deficit was still present. His thought process was slow, and most of the time he could not answer simple questions.

The cognitive function gradually improved. At 6,8 and 11 weeks post flupenthixol decanoate administration, the ECAQ scores were 6/11, 8/11 and $11 / 11$, respectively. Figure 1 illustrated the cognitive improvement experienced by the patient. At 7 weeks he developed parotitis secondary to the dry mouth and was managed by the otorhinolaryngologist. The patient refused antipsychotic treatment until 6 months later when he had a relapse. Oral olanzapine $2.5 \mathrm{mg}$ bis in die was started. The dosage was increased to $10 \mathrm{mg}$ omni nocte without any anticholinergic or extrapyramidal side effects.

\section{Discussion}

Flupenthixol, a thioxanthene, has an unarguably predominant antagonistic action at the dopamine receptors $(5,6)$. As for the anticholinergic property, literatures had contradicting view whether the anticholinergic effect is moderate (6) or clinically insignificant (5). At the beginning, when the patient presented with cognitive deficit and parkinsonism, anticholinergic medication was increased due to the impression that it was due to the excessive dopamine antagonist effects of flupenthixol decanoate and haloperidol. Haloperidol was discontinued and benzhexol dosage was increased. However, the cognition deteriorated further and he succumbed into a delirium state.

The dilemma faced by the clinician involved was due to the fact that the anticholinergic medication was indicated for the dopamine antagonist side effect but at the same time may cause a cognitive side effect. The administration of anticholinergic will improve the cognitive deficit due to dopamine blockade per se but may worsen it if the cause is via the anticholinergic mechanism. The administration of procyclidine helped clarifying the underlying mechanism. The sudden increase of anticholinergic activity after procyclidine injection resulted with an instant improvement of ECAQ score by 2. This is because the anticholinergic medication can indirectly increase the dopamine release into the synaptic cleft. The surge of 
dopamine neurotransmitter in the dorsolateral prefrontal cortex improves the cognition (1).

However, the improvement was not sustainable as procyclidine itself aggravated the existing anticholinergic adverse effect of flupenthixol, thus worsening the cognitive impairment.

The anticholinergic side effect was detrimental in this case. In the beginning, the benzhexol may contribute to the delirium state due to its combined effect with the flupenthixol. Considering its short elimination half-life of 3.7 hours, the washout period is estimated to be only around one day (7). Instead, the delirium and cognitive deficit persisted far beyond that point, thus suggesting it is highly likely due to the anticholinergic effect of flupenthixol decanoate rather than benzhexol.

To date, the literatures on anticholinergic property of flupenthixol did not specifically mention on the specific type of muscarinic receptors involved. However, from the apparent side effects of the patient, we postulated that the flupenthixol antagonized both central and peripheral muscarinic receptors. The $\mathrm{M}_{1}$ receptors are mainly responsible for both the executive function and submandibular gland secretion. Whereas the $\mathrm{M}_{3}$ are the main receptors for the intestinal motility as well as submandibular and parotid gland secretion (8).

The dopamine antagonist action was the least likely to be the main contributor in this case. Flupenthixol has a higher affinity towards $\mathrm{D}_{1}, \mathrm{D}_{2}$ and $D_{3}$ but less towards the $D_{4}$ receptors. $D_{2}$ receptors are abundantly found in the mesolimbic and nigrostriatal pathways that contribute to the psychosis and extrapyramidal side effects, respectively. Whereas the $\mathrm{D}_{4}$ receptors are mainly in the prefrontal cortex, thus they are related to the cognitive effect especially the executive function (2,9). Theoretically therefore, the extrapyramidal side effect is more likely to occur compared to impaired cognition based on the dopamine affinity of flupenthixol. Instead, parkinsonism did not recur after stopping the anticholinergic medication and continuous slow release of flupenthixol decanoate.

The patient most likely had an increased vulnerability towards severe anticholinergic side effect of flupenthixol. There were several factors behind this susceptibility. His age was the main contributing factor. Even in a non-diseased brain, the aging process may affect the structure of the muscarinic receptor thus decrease the acetylcholine volume and its receptors $(10,11)$.
This prompts the increased sensitivity towards acetylcholine. There was also an increased permeability of the blood-brain barrier and auxiliary leakages in old age especially those with neurodegenerative diseases (8). These permit the additional diffusion of flupenthixol particles into the brain and enhanced the anticholinergic repercussions. Schizophrenia itself is considered as a neurodegenerative disease with $60 \%$ showed an accelerated clinical deterioration despite being given the best treatment with favorable socioeconomic factor (12).

The intramuscular flupenthixol decanoate half-life is 17 days and the repeated administration ensures that the washout period to be approximately three months (6). It is meant to have a prolonged desired antipsychotic effect through each single administration but unfortunately so does the undesired adverse effect. In view that the flupenthixol is a lipophilic drug, its main disadvantage for the elderly is the prolongation of the half-life and the presence of more active free fractions of flupenthixol. This will accentuate the side effect properties in the elderly who is biologically having more fat, less water and less albumin compared to the body of their younger counterpart (6). The washout period did correlate with the patient improvement of the ECAQ score that resumed back to the perfect score at 11 weeks post administration. In view of the long period of washout, the patient needed a long hospital stay until the delirium resolved and the cognition adequate for an independent daily activities. There was no consensus regarding the antidote for anticholinergic delirium and anticholinergicinduced cognitive deficit up to date (10). However, a safe environment and supportive treatment are pivotal to prevent further complication. Fortunately, this condition is reversible.

In conclusion; A cognitive deficit with superimposed delirium in a treated schizophrenia patient may cause a confusion and dilemma in correctly determining the cause and delivering the best treatment. A wrong postulation can lead to a detrimental consequence in this otherwise reversible condition. Thus, psychiatrist and physician shall be a ware of these possible sequelae of flupenthixol in a susceptible individual to prevent further complication.

Acknowledgement: The authors would like to thank the Director General of Health Malaysia for his permission to publish this article. 


\section{References}

1. Ogino S, Miyamoto S, Miyake N, Yamaguchi N. Benefits and limits of anticholinergic use in schizophrenia: Focusing on its effect on cognitive function. Psychiatry Clin Neurosci 2014; 68: 37-49.

2. Meltzer HY, Park S, Kessler R. Cognition, schizophrenia, and the atypical antipsychotic drugs. PNAS 1999; 96: 13591-13593.

3. Elie D, Poirier M, Chianetta JM, Durand M, Grégoire CA, Grignon S. Cognitive effects of antipsychotic dosage and polypharmacy: a study with the BACS in patients with schizophrenia and schizoaffective disorder. J Psychopharmacol 2010; 24: 1037-1044.

4. Rehse M, Bartolovic M, Baum K, Richter D, Weisbrod M, Roesch-Ely D. Influence of antipsychotic and anticholinergic loads on cognitive functions in patients with schizophrenia. Schizophr Res Treatment 2016; 2016: 8213165.

5. Ownens DC. Meet the relatives: a reintroduction to the clinical pharmacology of 'typical' antipsychotics (Part 2). Adv Psychiatric Treatment 2012; 18: 337 350.

6. Taylor D, Paton C, Kapur S. The Maudsley Prescribing Guidelines in Psychiatry. 12th ed. West Sussex: Wiley Blackwell 2015.
7. Burke RE, Fahn S. Pharmacokinetics of trihexyphenidyl after short-term and long-term administration to dystonic patients. Ann Neurol 1985; 18: 35-40.

8. Abrams P, Andersson KE, Buccafusco JJ, Chapple C, de Groat WC, Fryer AD et al. Muscarinic receptors: their distribution and function in body systems, and the implications for treating overactive bladder. Br J Pharmacol 2006; 148: 565-578.

9. Mahapatra J, Quraishi SN, David A, Sampson S, Adams CE. Flupenthixol decanoate (depot) for schizophrenia or other similar psychotic disorders. Cochrane Database of Systematic Reviews 2014; CD001470.

10. Dawson AH, Buckley NA. Pharmacological management of anticholinergic delirium - theory, evidence and practice. Br J Clin Pharmacol 2015; 81: 516-524.

11. Ray PG, Meador KJ, Loring DW, Zamrini EW, Yang XH, Buccafusco JJ. Central anticholinergic hypersensitivity in aging. J Geriatr Psychiatry Neurol 1992; 5: 72-77.

12. McGlashan TH. The Chestnut Lodge follow-up study. II. Long-term outcome of schizophrenia and the affective disorders. Arch Gen Psychiatry 1984; 41: 586-601. 